Pacific Journal of Mathematics

ON THE HOMOTOPY INVARIANCE OF CERTAIN FUNCTOR 


\title{
ON THE HOMO'TOPY INVARIANCE OF CERTAIN FUNCTORS
}

\author{
BRIAN K. SCHMIDT
}

A functor from the category of topological spaces to the category of groups is said to be homotopy invariant if it carries homotopic mappings to the same mapping. It is well known, for example, that the homology and homotopy functors are homotopy invariant. On the other hand, the functor which takes each topological space $M$ to the free abelian group generated by the points of $M$ is not homotopy invariant. It will be shown that a functor which is not homotopy invariant must take topological spaces to groups which are very "large". For example, the homology groups of a simplicial complex are finitely generated, while the free abelian group generated by the points of a typical simplicial complex is uncountably generated. Among other results, it will be shown that every functor from simplicial complexes to finitely generated groups is homotopy invariant.

\section{Notation.}

1. Throughout this paper snt will denote the category of sets and $\mathfrak{T} \mathfrak{o p}$ will denote the category of topological spaces. We will denote by $\mathfrak{P}$ the full subcategory of $\mathfrak{I} \mathfrak{p p}$ whose objects are simplicial complexes. The closed interval $[0,1]$ on the real line will be denoted $I$, and the full subcategory of Iop whose only object is $I$ will be denoted Э. (S) will denote an arbitrary category.

We will use the word "functor" to mean a covariant functor and the word "cofunctor" to mean a contravariant functor. This allows us to say, for example, that homology is a functor and cohomology is a cofunctor. Thus a functor $\Omega: \Im \rightarrow(\$)$ assigns to the object $I$ in $\Im$ an object $\Omega(I)$ in $\mathbb{S}$ and to each continuous mapping $f: I \rightarrow I$ a morphism $\Omega(f): \Omega(I) \rightarrow \Omega(I)$ in such a way that composition and identity morphisms are preserved.

\section{Fundamentals.}

2. For each $x \in I$, let $k_{x}$ be the constant mapping from $I$ to $I$ which takes every element of $I$ to $x$.

3. TheOREm. Let $\Omega: \Im \rightarrow \Im$ be a functor. If there exist distinct continuous mappings $f, g: I \rightarrow I$ such that $\Omega(f)=\Omega(g)$, then there exist distinct $x, y \in I$ such that $\Omega\left(k_{x}\right)=\Omega\left(k_{y}\right)$. 
Proof. Since $f \neq g$, there exists $z \in I$ such that $f(z) \neq g(z)$. Let $x=f(z)$ and $y=g(z)$. Then $\Omega\left(k_{x}\right)=\Omega\left(k_{f(z)}\right)=\Omega\left(f k_{z}\right)=\Omega(f) \Omega\left(k_{z}\right)=$ $\Omega(g) \Omega\left(k_{z}\right)=\Omega\left(g k_{z}\right)=\Omega\left(k_{g(z)}\right)=\Omega\left(k_{y}\right)$.

4. THEOREM. Let $\Omega: \Im \rightarrow \&$ be a functor. If there exist distinct $x, y \in I$ such that $\Omega\left(k_{x}\right)=\Omega\left(k_{y}\right)$, then $\Omega\left(k_{0}\right)=\Omega\left(k_{1}\right)$.

Proof. It is clear that there exists a continuous mapping $f: I \rightarrow I$ such that $f(x)=0$ and $f(y)=1$. So $\Omega\left(k_{0}\right)=\Omega\left(k_{f(x)}\right)=\Omega\left(f k_{x}\right)=$ $\Omega(f) \Omega\left(k_{x}\right)=\Omega(f) \Omega\left(k_{y}\right)=\Omega\left(f k_{y}\right)=\Omega\left(k_{f(y)}\right)=\Omega\left(k_{1}\right)$.

5. THEOREM. If (S) is a category such that every functor $\Omega: \Im \rightarrow(S)$ satisfies $\Omega\left(k_{0}\right)=\Omega\left(k_{1}\right)$, then every functor $\Delta: \mathfrak{B} \rightarrow$ SS is homotopy invariant.

Proof. Let $f, g: M \rightarrow N$ be homotopic mappings in $\mathfrak{P}$. Define $j_{0}: M \rightarrow M \times I$ by $j_{0}(x)=(x, 0)$, and define $j_{1}: M \rightarrow M \times I$ by $j_{1}(x)=$ $(x, 1)$. Since $f$ and $g$ are homotopic, there exists a continuous mapping $h: M \times I \rightarrow N$ such that $f=h j_{0}$ and $g=h j_{1}$.

Define the functor $\Pi: \Im \rightarrow \Im$ by letting $\Pi(I)$ equal $M \times I$ and, for each continuous mapping $d: I \rightarrow I, \Pi(d)$ equal the mapping from $M \times I$ to $M \times I$ which takes $(x, y)$ to $(x, d(y))$. It is easy to verify that $\Pi\left(k_{0}\right) j_{0}=j_{0}$ and $\Pi\left(k_{1}\right) j_{0}=j_{1}$. And $\Delta \Pi: \mathfrak{\Im} \rightarrow \mathbb{B S}$ is a functor, so $\Delta \Pi\left(k_{0}\right)=\Delta \Pi\left(k_{1}\right) . \quad$ Thus $\quad \Delta(f)=\Delta\left(h j_{0}\right)=\Delta(h) \Delta\left(j_{0}\right)=\Delta(h) \Delta\left(\Pi\left(k_{0}\right) j_{0}\right)=$ $\Delta(h) \Delta \Pi\left(k_{0}\right) \Delta\left(j_{0}\right)=\Delta(h) \Delta \Pi\left(k_{1}\right) \Delta\left(j_{0}\right)=\Delta(h) \Delta\left(\Pi\left(k_{1}\right) j_{0}\right)=\Delta(h) \Delta\left(j_{1}\right)=\Delta\left(h j_{1}\right)=$ $\Delta(g)$.

6. THEOREM. If for every functor $\Omega: \Im \rightarrow \$$ there exist distinct continuous mappings $f, g: I \rightarrow I$ such that $\Omega(f)=\Omega(g)$, then every functor $\Delta: \mathfrak{P} \rightarrow \mathbb{B S}$ is homotopy invariant.

Proof. Combine Theorems 3, 4, and 5.

7. All of our results on homotopy invariance will be based on Theorem 6. To get these results, we must find methods of showing that for certain categories $\mathbb{S}$, every functor $\Omega: \mathfrak{\Im} \rightarrow \$ S$ takes two distinct continuous mappings to the same morphism. We will present several methods, each applicable to a certain type of category. Our first method is quite simple.

First Approach - Functors.

8. THEOREM. Let (8) be a category such that for every object 
$G$ in (S) the set of morphisms from $G$ to $G$ is countable. Then every functor $\Delta: \mathfrak{P} \rightarrow \mathbb{B S}$ is homotopy invariant.

Proof. Consider any functor $\Omega: \mathfrak{\Im} \rightarrow$ BS. The set of morphisms from $\Omega(I)$ to $\Omega(I)$ is countable, but the set of continuous mappings from $I$ to $I$ is uncountable. Hence there exist distinct continuous mappings $f, g: I \rightarrow I$ such that $\Omega(f)=\Omega(g)$.

9. For example, every functor from $\mathfrak{F}$ to the category of finitely generated abelian groups must be homotopy invariant. Thus Theorem 8 constitutes a proof that the homology functors, with domain $\mathfrak{P}$, are homotopy invariant. This is rather surprising, since we have made very little use of the definition of homology. Similarly, every functor from $\mathfrak{P}$ to the category of finitely generated groups must be homotopy invariant. This proves that the first homotopy functor $\pi_{1}$, with domain $\mathfrak{P}$, is homotopy invariant. And Theorem 8 can be applied to many other categories whose objects are finite or finitely generated in some sense.

\section{First Approach - Cofunctors.}

10. Now we will turn to cofunctors. In view of the fact that a cofunctor to $(3)$ is the same thing as a functor to (3), where (3) is the category dual to (S), Theorems 6 and 8 may be restated to deal with cofunctors as follows:

11. THEOREM. If for every cofunctor $\Omega: \Im \rightarrow$ (S) there exist distinct continuous mappings $f, g: I \rightarrow I$ such that $\Omega(f)=\Omega(g)$, then every cofunctor $\Delta: \mathfrak{S} \rightarrow$ BS is homotopy invariant.

12. THEOREM. Let (S) be a category such that for every object $G$ in (S) the set of morphisms from $G$ to $G$ is countable. Then every cofunctor $\Delta: \mathfrak{B} \rightarrow(S$ is homotopy invariant.

13. For example, every cofunctor from $\mathfrak{P}$ to the category of finitely generated abelian groups must be homotopy invariant. This proves that the cohomology cofunctors are homotopy invariant. Here is a more unusual example. A functor or cofunctor $\Delta: \mathfrak{P} \rightarrow \mathfrak{P}$ is said to be homotopy preserving if, for any two homotopic mappings $f, g: M \rightarrow N$ in $\mathfrak{P}, \Delta(f)$ is homotopic to $\Delta(g)$.

14. THEOREM. Every functor or cofunctor $\Delta: \mathfrak{P} \rightarrow \mathfrak{P}$ is homotopy preserving.

Proof. Let $\mathfrak{P h}$ denote the category whose objects are simplicial 
complexes and whose morphisms are homotopy classes of continuous mappings. Let $\Phi: \mathfrak{P} \rightarrow \mathfrak{P G}$ be the functor given by $\Phi(M)=(M)$ and $\Phi(f)=$ the homotopy class of $f$. Given any simplicial complex $M$, the simplicial approximation theorem says that every continuous mapping from $M$ to $M$ is homotopic to a simplicial mapping. And the set of simplicial mappings from $M$ to $M$ is obviously countable. Hence the set of morphisms from $M$ to $M$ in $\mathfrak{P h}$ is countable. Thus, by Theorems 8 and 12 , every functor or cofunctor from $\mathfrak{P}$ to $\mathfrak{P h}$ is homotopy invariant. In particular, $\Phi \Delta: \mathfrak{P} \rightarrow \mathfrak{P h}$ is homotopy invariant. And this says precisely that $\Delta$ is homotopy preserving.

\section{Generalizations.}

15. We will say that a category $\mathfrak{I}$ is "admissible" if Theorems $6,8,11$, and 12 remain true when $\mathfrak{P}$ is replaced by $\mathfrak{T}$. For example, let $\mathfrak{I}$ be a full subcategory of $\mathfrak{I} \mathrm{Dp}$. It is clear that the proofs of these theorems stand without modification provided that $I$ is an object of $\mathfrak{I}$ and $\mathfrak{I}$ is closed under the operation product-with- $I$. Hence all such categories are admissible. Moreover, $\mathfrak{I}$ may be admissible even if $\mathfrak{I}$ is not closed under product-with- $I$ or if $I$ is not an object of $\mathfrak{I}$. For if the real line $\boldsymbol{R}$ is an object of $\mathfrak{T}$ and $\mathfrak{T}$ is closed under productwith- $\boldsymbol{R}$, we may replace $I$ by $\boldsymbol{R}$ in the proofs of $6,8,11,12$, and all preceding theorems. This does not change the meaning of homotopy: Two continuous mappings $f, g: M \rightarrow N$ are homotopic in the usual sense if and only if there exists a continuous mapping $h: M \times R \rightarrow N$ such that $h(x, 0)=f(x)$ and $h(x, 1)=g(x)$ for all $x \in M$. Hence the category of topological manifolds with boundary is admissible, even though it is not closed under product-with- $I$. And the category of topological manifolds is admissible, though it does not contain the object $I$. Similarly, if the circle $S^{1}$ is an object of $\mathfrak{I}$ and $\mathfrak{I}$ is closed under product-with- $S^{1}$, then $\mathfrak{I}$ is admissible. Hence the category of compact topological manifolds and the category of compact topological manifolds with boundary are admissible. In general, given a topological space $H$, distinct points $y, z \in H$, and continuous mappings $f, g: M \rightarrow N$, we say that $f$ and $g$ are $(H, y, z)$-homotopic if there exists a continuous mapping $h: M \times H \rightarrow N$ such that $h(x, y)=f(x)$ and $h(x, z)=g(x)$ for all $x \in M$. It is not hard to verify that $(H, y, z)$-homotopy is equivalent to ordinary homotopy if

(a) there exist continuous mappings $i: I \rightarrow H, j: H \rightarrow I$ such that $i(0)=y, i(1)=z, j(y)=0$, and $j(z)=1$.

And the proof of Theorem 4 remains valid if

(b) given distinct $s, t \in H$, there exists a continuous mapping $m: H \rightarrow H$ such that $m(s)=y$ and $m(t)=z$.

Thus, if (a) and (b) are satisfied, every category $\mathfrak{I}$ which contains 
$H$ and is closed under product-with- $H$ is admissible.

16. There are some admissible subcategories of Iop which are not full. Consider, for example, the category of smooth manifolds. As was said in 15, two continuous mappings $f, g: M \rightarrow N$ are homotopic if and only if there exists a continuous mapping $h: M \times R \rightarrow N$ such that $h(x, 0)=f(x)$ and $h(x, 1)=g(x)$. And it is well known that if $f$ and $g$ are smooth, we may take $h$ to be smooth. (Smooth homotopy is equivalent to continuous homotopy.) Thus, replacing $I$ by $R$, we find that the category of smooth manifolds is admissible. Similarly, replacing $I$ by $\boldsymbol{R}$ or $S^{1}$, we find that the following categories are admissible: smooth manifolds with boundary, compact smooth manifolds, compact smooth manifolds with boundary.

17. Let $\mathfrak{B}_{2}$ denote the category of pairs of simplicial complexes. An object in $\mathfrak{P}_{2}$ is a pair $\left(M_{1}, M_{2}\right)$, where $M_{1}$ and $M_{2}$ are simplicial complexes and $M_{2}$ is a subset of $M_{1}$. And a morphism $f:\left(M_{1}, M_{2}\right) \rightarrow$ $\left(N_{1}, N_{2}\right)$ is a continuous mapping from $M_{1}$ to $N_{1}$ such that $f\left(M_{2}\right) \subset N_{2}$. We say that two morphisms $f, g:\left(M_{1}, M_{2}\right) \rightarrow\left(N_{1}, N_{2}\right)$ are homotopic if and only if there exists a continuous mapping $h: M_{1} \times I \rightarrow N_{1}$ such that $h(x, 0)=f(x)$ and $h(x, 1)=g(x)$ for all $x \in M$, and $h\left(M_{2}, y\right) \subset N_{2}$ for all $y \in I$. If we replace $I$ by the pair $(I, I)$ in the proofs of $6,8,11,12$, and all preceding theorems, it follows that $\mathfrak{F}_{2}$ is an admissible category. Likewise, the category of pairs of any category in 15 or 16 is admissible.

\section{A Generalization That Fails.}

18. Since the cardinality of $I$ is $C$, the cardinality of the continuum, one would expect the set of continuous mappings from $I$ to $I$ to have cardinality greater than $C$. But a continuous mapping from $I$ to $I$ is determined by its restriction to the set of rational numbers in the domain. Hence the set of continuous mappings from $I$ to $I$ has cardinality $C$. Thus the proof of Theorem 8 does not work for categories (3) in which the set of morphisms from $G$ to $G$ can have cardinality $C$. We will now strengthen Theorem 8 to make it work for many such categories.

\section{Second Approach - Functors.}

19. Let $B$ be a set, and let $\mathfrak{B}$ be the full subcategory of (5ns whose only object is $B$. Suppose there exists a functor $\Omega: \mathfrak{\Im} \rightarrow \mathfrak{B}$ such that $\Omega(f)=\Omega(\mathrm{g})$ only if $f=g$. We will prove that $B$ is uncountable. As in 2, let $k_{x}: I \rightarrow I$ be the mapping which takes every element of $I$ to $x$. Let $e_{x}=\Omega\left(k_{x}\right)$. 
20. THEOREM. For all $x, y \in I, e_{y} e_{x}=e_{y}$.

Proof. $\quad e_{y} e_{x}=\Omega\left(k_{y}\right) \Omega\left(k_{x}\right)=\Omega\left(k_{y} k_{x}\right)=\Omega\left(k_{y}\right)=e_{y}$.

21. CoRollary. For all $x \in I, e_{x} e_{x}=e_{x}$.

22. THEOREM. Consider $b \in B$ and distinct $x, y \in I$. If $e_{x}(b)=$ $e_{y}(b)$, then $e_{z}(b)=e_{x}(b)$ for all $z \in I$.

Proof. Clearly there exists a continuous mapping $f: I \rightarrow I$ such that $f(x)=x$ and $f(y)=z$. Then $f k_{x}=k_{x}$ and $f k_{y}=k_{z}$. 'So $Q(f) e_{x}=$ $\Omega(f) \Omega\left(k_{x}\right)=\Omega\left(f k_{x}\right)=\Omega\left(k_{x}\right)=e_{x}$. Likewise, $\Omega(f) e_{y}=e_{z}$. Thus $e_{z}(b)=$ $\Omega(f) e_{y}(b)=\Omega(f) e_{x}(b)=e_{x}(b)$.

23. Let $B_{0}$ be the set of all $b \in B$ such that $e_{x}(b)=b$ for all $x \in I$. And let $B_{1}$ be the set of all $b \in B$ such that $e_{x}(b)=b$ for exactly one $x \in I$.

24. Corollary. Consider $b \in B$ and distinct $x, y \in I$. If $e_{x}(b)=b$ and $e_{y}(b)=b$, then $b \in B_{0}$.

25. Corollary. Consider $b \in B$ and $x \in I$. If $e_{x}(b)=b$, then either $b \in B_{0}$ or $b \in B_{1}$.

26. Theorem. $B_{1}$ is not empty.

Proof. Suppose $B_{1}=\varnothing$. Consider any $b \in B$ and distinct $x, y \in I$. By Corollary 21, $e_{x}\left(e_{x}(b)\right)=e_{x}(b)$. Hence, by Corollary 25, $e_{x}(b)$ is an element of either $B_{0}$ or $B_{1}$. Since $B_{1}=\varnothing, e_{x}(b) \in B_{0}$. Thus $e_{y}\left(e_{x}(b)\right)=$ $e_{x}(b)$. But by Theorem 20, $e_{y}\left(e_{x}(b)\right)=e_{y}(b)$. Since $b$ was arbitrary, we have $e_{x}=e_{y}$, or $\Omega\left(k_{x}\right)=\Omega\left(k_{y}\right)$. This contradicts 19 .

27. Let $c$ be any element of $B_{1}$. Define a mapping $\omega: I \rightarrow B$ by $\omega(x)=e_{x}(c)$.

28. THEOREM. $\omega$ is one-to-one.

Proof. Suppose that there exist distinct $x, y \in I$ such that $\omega(x)=$ $\omega(y)$; that is, $e_{x}(c)=e_{y}(c)$. Then Theorem 22 says that $e_{z}(c)$ must be the same element of $B$ for all $z \in I$. But there is exactly one $z \in I$ such that $e_{z}(c)=c$, since $c \in B_{1}$. This is a contradiction.

29. CoRollary. If $B$ is a set as in 19, then $B$ is uncountable.

30. TheOREm. Let (S) be a subcategory of (Fns in which every 
object is countable. Then every functor $\triangle: \mathfrak{P} \rightarrow \mathbb{B S}$ is homotopy invariant.

Proof. Consider any functor $\Omega: \Im \rightarrow$ S. Let $B=\Omega(I)$, and let $\mathfrak{B}$ denote the full subcategory of $\mathfrak{n} n \mathrm{~s}$ whose only object is $B$. We may view $\Omega$ as a functor from $\Im$ to $\mathfrak{B}$. Since $B$ is countable, Corollary 29 says that there exist distinct continuous mappings $f, g: I \rightarrow I$ such that $\Omega(f)=\Omega(g)$. Hence, by Theorem 6 , every functor $\Delta: \mathfrak{P} \rightarrow \mathbb{B}$ is homotopy invariant.

31. For example, every functor from $\mathfrak{P}$ to the category of countable abelian groups must be homotopy invariant. This proves that the $n$th homotopy functor $\pi_{n}$, with domain $\mathfrak{F}$, is homotopy invariant. Similarly, every functor from $\mathfrak{P}$ to the category of countable rings must be homotopy invariant.

32. For completeness, we will prove two more theorems at this point. Let $B, \mathfrak{B}$, and $\Omega$ be as in 19 .

33. Theorem. $\omega(x) \in B_{1}$, for all $x \in I$.

Proof. For any $y \in I, e_{y} \omega(x)=e_{y} e_{x}(c)=e_{y}(c)=\omega(y)$. Thus $e_{y} \omega(x)=$ $\omega(x)$ precisely when $y=x$.

34. Theorem. For any continuous mapping $f: I \rightarrow I, \Omega(f) \omega=\omega f$.

Proof. For any $x \in I, \Omega(f) \omega(x)=\Omega(f) e_{x}(c)=\Omega(f) \Omega\left(k_{x}\right)(c)=\left(f k_{x}\right)(c)=$ $\Omega\left(k_{f(x)}\right)(c)=e_{f(x)}(c)=\omega f(x)$.

35. This theorem asserts the naturality of $\omega$. Strictly speaking, it says that $\omega$ is a natural transformation from the forgetful functor (from $\Im$ to (n? $)$ to $\Omega$ (viewed as a functor from $\Im$ to (5ng). [1].

\section{Second Approach - Cofunctors.}

36. Now we would like to prove that Theorem 30 remains true if $\Delta$ is a cofunctor instead of a functor. Unfortunately, the proof of Theorem 30 relies on the construction of a mapping $\omega: I \rightarrow B$, and this construction does not work for cofunctors. Hence we must take a slightly different approach.

37. As before, let $B$ be a set and let $\mathfrak{B}$ be the full subcategory of rits whose only object is $B$. Suppose there exists a cofunctor $\Omega: \mathfrak{\Im} \rightarrow \mathfrak{B}$ such that $\Omega(f)=\Omega(g)$ only if $f=g$. 
38. Recall that $I$ is a linearly ordered set. Given $x, y \in I$, let $p_{x}(y)$ denote the smaller of $x$ and $y$. Then $p_{x}: I \rightarrow I$ is a continuous mapping. And let $\mathfrak{2 H A t}_{+}$denote the set of all continuous mappings $f: I \rightarrow I$ such that $f^{-1}: I \rightarrow I$ exists $\left(f^{-1} f\right.$ and $f f^{-1}$ are the identity) and such that $x<y$ implies $f(x)<f(y)$. It is easy to verify that:

39. If $x \leqq y$, then $p_{x} p_{y}=p_{x}$ and $p_{y} p_{x}=p_{x}$.

40. Given $x \in I$ and $f \in \mathfrak{P H t}_{+}, f p_{x} f^{-1}=p_{f(x)}$.

Let $q_{x}=\Omega\left(p_{x}\right)$.

41. THEOREM. If $x \leqq y$, then $q_{x} q_{y}=q_{x}$ and $q_{y} q_{x}=q_{x}$.

Proof. Using 39, we have $q_{x} q_{y}=\Omega\left(p_{x}\right) \Omega\left(p_{y}\right)=\Omega\left(p_{y} p_{x}\right)=\Omega\left(p_{x}\right)=$ $q_{x}$. Likewise $q_{y} q_{x}=\Omega\left(p_{y}\right) \Omega\left(p_{x}\right)=\Omega\left(p_{x} p_{y}\right)=\Omega\left(p_{x}\right)=q_{x}$.

42. Theorem. Given $x \in I$ and $f \in \mathfrak{Q H n t}_{+}, \Omega\left(f^{-1}\right) q_{x} \Omega(f)=q_{f(x)}$.

Proof. Using 40, we have

$$
\Omega\left(f^{-1}\right) q_{x} \Omega(f)=\Omega\left(f^{-1}\right) \Omega\left(p_{x}\right) \Omega(f)=\Omega\left(f p_{x} f^{-1}\right)=\Omega\left(p_{f(x)}\right)=q_{f(x)} .
$$

43. TheOREM. For any $f \in \mathfrak{U H}_{+}, \Omega\left(f^{-1}\right)=[\Omega(f)]^{-1}$.

Proof. Let $1_{I}$ and $1_{B}$ denote the identity mappings on $I$ and $B$ respectively. Then $\Omega(f) \Omega\left(f^{-1}\right)=\Omega\left(f^{-1} f\right)=\Omega\left(1_{I}\right)=1_{B}$. Likewise $\Omega\left(f^{-1}\right) \Omega(f)=\Omega\left(f f^{-1}\right)=\Omega\left(1_{I}\right)=1_{B}$.

44. Define a mapping $\lambda: B \rightarrow I$ by letting $\lambda(b)$ equal the greatest lower bound in $I$ of $\left\{x \in I \mid q_{x}(b)=b\right\}$. Note that we do not know whether $q_{\lambda(b)}(b)=b$. But we can at least say the following.

45. Theorem. Consider $x \in I$ and $b \in B$ with $x \neq \lambda(b)$. Then $\lambda(b)<x$ if and only if $q_{x}(b)=b$.

Proof. If $q_{x}(b)=b$, it is obvious that $\lambda(b)<x$. Conversely, if $\lambda(b)<x$, there exists $w \in I$ such that $\lambda(b) \leqq w<x$ and $q_{w}(b)=b$. By Theorem $41, q_{x} q_{w}=q_{w}$. Thus $q_{x} q_{w}(b)=q_{w}(b)$, which says that $q_{x}(b)=b$.

46. ThEOREM. For any $f \in \mathfrak{U}_{\mathfrak{H t t}}^{+}, f^{-1} \lambda=\lambda \Omega(f)$.

Proof. Consider $x \in I$ and $b \in B$ such that $x$ is not equal to $f^{-1} \lambda(b)$ or $\lambda \Omega(f)(b)$. It suffices to show that $f^{-1} \lambda(b)<x$ if and only if $\lambda \Omega(f)(b)<x$. Our approach will be the following. 


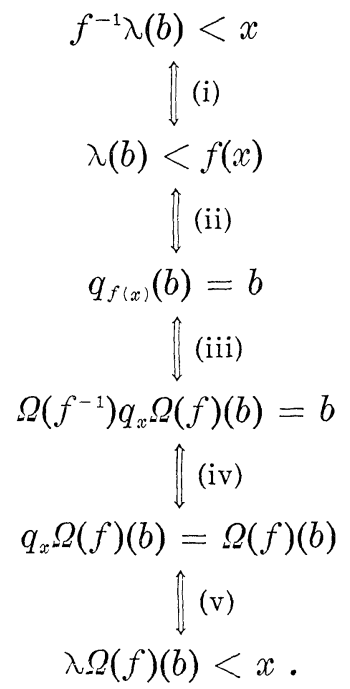

(i) follows from the fact that $f$ and $f^{-1}$ belong to $\mathfrak{Q u t}_{+}$. Note that, since $x \neq f^{-1} \lambda(b)$, we have $f(x) \neq \lambda(b)$. Thus (ii) follows from Theorem 45. (iii) follows from Theorem 42, and (iv) follows from Theorem 43. And, since we have assumed that $x \neq \lambda \Omega(f)(b)$, (v) follows from Theorem 45.

47. This theorem asserts a kind of naturality of $\lambda$. Viewing $\mathfrak{R u t}_{+}$as a category whose only object is $I$, we may define a cofunctor $\Lambda: \mathfrak{Q U t u}_{+} \rightarrow$ Snte by letting $\Lambda(I)=I$ and $\Lambda(f)=f^{-1}$. Then $\lambda$ is a natural transformation from $\Omega$ (viewed as a cofunctor from $\mathfrak{W h t}_{+}$to (5nt) to $\Lambda$. Note that this kind of naturality is much more restricted than that of Theorem 34 .

48. Theorem. There exists $c \in B$ such that $0<\lambda(c)<1$.

Proof. Suppose there is no such $c$. By Theorem 41, $q_{1 / 2}\left(q_{1 / 2}(b)\right)=$ $q_{1 / 2}(b)$ for all $b \in B$. Thus by Theorem $45, \lambda q_{1 / 2}(b) \leqq 1 / 2$. So $\lambda q_{1 / 2}(b)$ must equal 0 . Therefore, by 45 again, $q_{1 / 4}\left(q_{1 / 2}(b)\right)=q_{1 / 2}(b)$ for all $b \in B$. But, by Theorem 41, $q_{1 / 4}\left(q_{1 / 2}(b)\right)=q_{1 / 4}(b)$ for all $b \in B$. So $q_{1 / 2}=q_{1 / 4}$, which says that $\Omega\left(p_{1 / 2}\right)=\Omega\left(p_{1 / 4}\right)$. This contradicts 37 .

49. Theorem. $\lambda$ maps $B$ onto the interior of $I$.

Proof. By Theorem 48, there exists $c \in B$ with $0<\lambda(c)<1$. Given any $x \in I$ with $0<x<1$, there exists $f \in \mathfrak{N H t}_{+}$such that $f^{-1} \lambda(c)=x$. Thus, by Theorem $46, \lambda(\Omega(f)(c))=x$.

50. Corollary. If $B$ is a set as in 37 , then $B$ is uncountable. 
51. TheOREM. Let (S) be a subcategory of Eng in which every object is countable. Then every cofunctor $\Delta: \mathfrak{B} \rightarrow \mathbb{B}$ is homotopy invariant.

Proof. Corollary 50 says that for every cofunctor $\Omega: \Im \rightarrow(S$ there exist distinct continuous mappings $f, g: I \rightarrow I$ such that $\Omega(f)=$ $\Omega(g)$. Hence the assertion follows by Theorem 11 .

\section{Generalizations.}

52. In Theorem $30, \mathfrak{P}$ may be replaced by any of the categories named in 15,16 or 17 . If we replace $I$ by a space $H$ as in 15 , the proof of Theorem 22 requires that $H$ satisfy the added condition that

(c) given distinct $s, t \in H$ and any $u \in H$, there exists a continuous mapping $m: H \rightarrow H$ such that $m(s)=s$ and $m(t)=u$. This is satisfied by $R, S^{1}$, and many other spaces.

In Theorem $51, \mathfrak{P}$ may be replaced by any of the categories named in 15 or 17 . We may replace $I$ by $\boldsymbol{R}$ or $S^{1}$ as in 15 , but our construction of $\lambda$ makes it difficult to replace $I$ by anything more general. Likewise, the construction of $\lambda$ rules out the categories in 16 , which admit only smooth mappings. This happens because the mappings $p_{x}: I \rightarrow I$ have no smooth analog.

53. Note that the proof of Theorem 51 is very symmetrical. In particular, this proof can be daulized to give a proof of Theorem 30 . But the proof we have given of Theorem 30 is simpler and more general, in that it applies to smooth structures as well as topological structures.

54. There is one important category (s) to which Theorems 30 and 51 do not apply: the category $\mathfrak{B}_{K}^{o}$ of countable dimensional vector spaces over a field $K$. The problem is that a countable dimensional vector space (or even a finite dimensional vector space) may have an uncountable number of elements. It will now be shown that Theorems 30 and 51 remain true when $\mathbb{B S}=\mathfrak{B}_{K}^{o}$.

55. THEOREM. Every functor $\triangle: \mathfrak{P} \rightarrow \mathfrak{B}_{K}^{\sigma}$ is homotopy invariant.

Proof. We will proceed as in Theorems 20-29. Let $B$ be a vector space over $K$, and let $\mathfrak{B}$ be the full subcategory of the category of vector spaces over $K$ whose only object is $B$. If there exists a functor $\Omega: \Im \rightarrow \mathfrak{B}$ such that $\Omega(f)=\Omega(g)$ only if $f=g$, then there is a oneto-one mapping $\omega: I \rightarrow B$ such that $\Omega(f) \omega=\omega f$ for all continuous mappings $f: I \rightarrow I$. Suppose that the image of $\omega$ is a linearly dependent 
set. Then there exists an element $x \in I$, a finite set $Y \subset I-\{x\}$, and scalars $a_{y} \in K$ indexed by $y \in Y$ such that $\omega(x)=\sum_{y \in Y} a_{y} \omega(y)$. It is easy to construct a continuous mapping $f: I \rightarrow I$ such that $f(y)=y$ for all $y \in Y$ and $f(x) \neq x$. Then

$$
\begin{aligned}
\omega(f(x)) & =\Omega(f) \omega(x)=\Omega(f)\left(\sum_{y \in Y} a_{y} \omega(y)\right)=\sum_{y \in Y} a_{y} Q(f) \omega(y) \\
& =\sum_{y \in Y} a_{y} \omega(f(y))=\sum_{y \in Y} a_{y} \omega(y)=\omega(x) .
\end{aligned}
$$

This is a contradiction, since $\omega$ is one-to-one. Hence the image of $\omega$ is a linearly independent set. And the image of $\omega$ is uncountable, so it follows that the dimension of $B$ is uncountable. Thus for every functor $\Omega: \mathfrak{J} \rightarrow \mathfrak{B}_{K}^{o}$ there exist distinct continuous mappings $f, g: I \rightarrow I$ such that $Q(f)=Q(g)$. Therefore, by Theorem 6, every functor $\Delta: \mathfrak{B} \rightarrow \mathfrak{B}_{K}^{\sigma}$ is homotopy invariant.

56. THEOREM. Every cofunctor $\Delta: \mathfrak{P} \rightarrow \mathfrak{S}_{K}^{\sigma}$ is homotopy invariant.

Proof. This is analogous to the proof of Theorem 55. Proceed as in Theorems $41-50$, and use naturality of $\lambda$.

57. Note that we may replace $\mathfrak{B}_{K}^{\sigma}$ by the category $\mathfrak{I}_{K}^{o}$ of countable dimensional algebras over $K$ in Theorems 55 and 56 , because every functor $\Delta: \mathfrak{B} \rightarrow \mathfrak{X}_{K}^{\sigma}$ can be viewed as a functor from $\mathfrak{i}$ to $\mathfrak{I}_{K}^{\sigma}$.

Conclusion.

List A: simplicial complexes

topological manifolds

topological manifolds with boundary

compact topological manifolds

compact topological manifolds with boundary

pairs in any category above

List B: smooth manifolds

smooth manifolds with boundary

compact smooth manifolds

compact smooth manifolds with boundary

pairs in any category above

List C: finitely generated abelian groups

finitely generated groups

finitely generated rings

List D: countable abelian groups

countable groups

countable rings 


\section{countable dimensional vector spaces over a field $K$} countable dimensional algebras over a field $K$.

58. We have shown that every functor from a category in List $\mathrm{A}$ or $\mathrm{B}$ to a category in List $\mathrm{C}$ or $\mathrm{D}$ is homotopy invariant. And every cofunctor from a category in List $A$ to a category in List C or $\mathrm{D}$, or from a category in List $\mathrm{B}$ to a category in List $\mathrm{C}$, is homotopy invariant. And these results can be extended to many other categories by the methods developed herein. Some questions that remain are:

1. Is every cofunctor from smooth manifolds to countable groups homotopy invariant?

2. For which rings $R$ can the category of countably generated $R$-modules be placed in List $\mathrm{D}$ ?

\section{REFERENCES}

1. Peter Freyd, Abelian Categories, Harper and Row, 1964.

2. John W. Keesee, On the homotopy axiom, Ann. of Math., (2) 54 (1951), 247-249.

3. James R. Munkres, Elementary Differential Topology, Princeton University Press, 1966.

4. Edwin H. Spanier, Algebraic Topology, McGraw-Hill Book Co., 1966.

Received February 21, 1973.

SOUTHERN ILlinoIS UNIVERSity 


\section{PACIFIC JOURNAL OF MATHEMATICS}

\section{EDITORS}

RICHARD ARENS (Managing Editor)

University of California

Los Angeles, California 90024

R. A. Beaumont

University of Washington

Seattle, Washington 98105
J. DugundJI

Department of Mathematics University of Southern California Los Angeles, California 90007

D. Gilbarg AND J. Milgram Stanford University

Stanford, California 94305

\section{ASSOCIATE EDITORS}
E. F. BECKENBACH
B. H. NeUmanN
F. WOLF
K. YOSHIDA

\section{SUPPORTING INSTITUTIONS}

UNIVERSITY OF BRITISH COLUMBIA CALIFORNIA INSTITUTE OF TECHNOLOGY

UNIVERSITY OF CALIFORNIA

MONTANA STATE UNIVERSITY

UNIVERSITY OF NEVADA

NEW MEXICO STATE UNIVERSITY

OREGON STATE UNIVERSITY

UNIVERSITY OF OREGON

OSAKA UNIVERSITY
UNIVERSITY OF SOUTHERN CALIFOF

STANFORD UNIVERSITY

UNIVERSITY OF TOKYO

UNIVERSITY OF UTAH

WASHINGTON STATE UNIVERSITY UNIVERSITY OF WASHINGTON

AMERICAN MATHEMATICAL SOCIET NAVAL WEAPONS CENTER 


\section{Pacific Journal of Mathematics}

\section{Vol. 54, No. 1 \\ May, 1974}

Ralph K Amayo, Engel Lie rings with chain conditions ..................

Bernd Anger and Jörn Lembcke, Hahn-Banach type theorems for hypolinear

functionals on preordered topological vector spaces ..................

Gregory Frank Bachelis and Samuel Ebenstein, On $\Lambda(p)$ sets ................

Harvey Isaac Blau, Indecomposable modules for direct products of finite

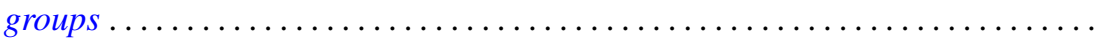

Larry Eugene Bobisud and James Calvert, Singular perturbation of a

time-dependent Cauchy problem in a Hilbert space ................

Walter D. Burgess and Robert Raphael, Abian's order relation and orthogonal

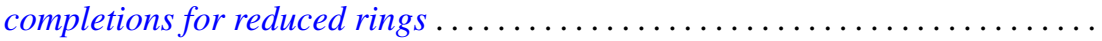

James Diederich, Representation of superharmonic functions mean continuous at

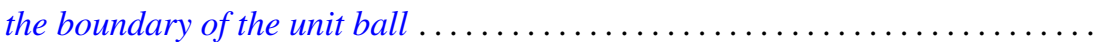

Aad Dijksma and Hendrik S. V. de Snoo, Self-adjoint extensions of symmetric

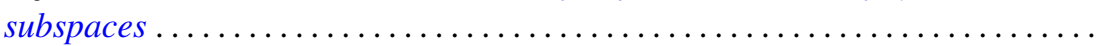

Gustave Adam Efroymson, A Nullstellensatz for Nash rings . . . . . . . . . . . . .

John D. Elwin and Donald R. Short, Branched immersions onto compact orientable surfaces . . . . . . . . . . . . . . . . . . . . . . . . .

John Douglas Faires, Comparison of the states of closed linear

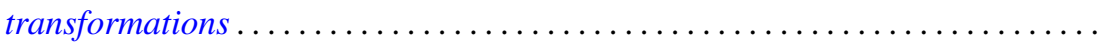

Joe Wayne Fisher and Robert L. Snider, On the von Neumann regularity of rings with regular prime factor rings .

Franklin Takashi Iha, A unified approach to boundary value problems on compact intervals

Palaniappan L. Kannappan and Che Tat $\mathrm{Ng}$, On functional equations connected with directed divergence, inaccuracy and generalized directed divergence

Samir A. Khabbaz and Elias Hanna Toubassi, The module structure of Ext $(F, T)$ over the endomorphism ring of $T$...

Garo K. Kiremidjian, On deformations of complex compact manifolds with boundary.

Dimitri Koutroufiotis, Mappings by parallel normals preserving principal

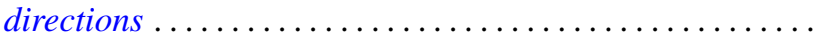

W. K. Nicholson, Semiperfect rings with abelian adjoint group

Norman R. Reilly, Extension of congruences and homomorphisms to translational hulls

Sadahiro Saeki, Symmetric maximal ideals in $M(G)$

Brian Kirkwood Schmidt, On the homotopy invariance of certain functors ...

H. J. Shyr and T. M. Viswanathan, On the radicals of lattice-ordered rings ...

Indranand Sinha, Certain representations of infinite group algebras ...

David Smallen, The group of self-equivalences of certain complexes ...

Kalathoor Varadarajan, On a certain problem of realization in homotopy

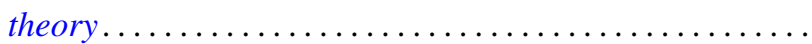

